\title{
Probabilistic Modelling of Target Drift in Marine Disasters Using Ocean Currents From High- Frequency Radars
}

\section{Kui Zhu}

Naval University of Engineering

Lin Mu ( $\nabla$ zeek@cug.edu.cn )

China University of Geosciences

Haiwen Tu

China University of Geosciences

Runze Yu

China Petroleum Pipeline Engineering Corporation

Xiaoyu Xia

China University of Geosciences

\section{Research Article}

Keywords: Target Drift Prediction, Search and Rescue, Sub-grid scale velocity model, Diffusion, HighFrequency Radar

Posted Date: February 16th, 2022

DOI: https://doi.org/10.21203/rs.3.rs-1344742/v1

License: (c) (1) This work is licensed under a Creative Commons Attribution 4.0 International License. Read Full License 


\title{
Probabilistic modelling of target drift in marine disasters using
} ocean currents from high-frequency radars

\author{
Kui Zhu ${ }^{\mathrm{a}}$, Lin $\mathrm{Mu}^{\mathrm{b}, \mathrm{c}^{*}}$, Haiwen $\mathrm{Tu}^{\mathrm{b} *}$, Runze Yud ${ }^{\mathrm{d}}$, Xiaoyu Xia ${ }^{\mathrm{b}}$ \\ ${ }^{a}$ College of Electrical Engineering, Naval University of Engineering, Wuhan \\ 430033, PR China \\ ${ }^{\mathrm{b}}$ College of Marine Science and Technology, China University of Geosciences, \\ Wuhan 430074, PR China \\ ${ }^{\mathrm{c}}$ College of Life Sciences and Oceanography, Shenzhen University, Shenzhen \\ 518060, PR China \\ d. China Petroleum Pipeline Engineering Corporation,Langfang 065000,PR China \\ Correspondence: Lin Mu (cug.zk@foxmail.com)
}

\begin{abstract}
College of Electrical Engineering, Naval University of Engineering, Wuhan 430033, PR China (zeek@cug.edu.cn)
\end{abstract}

\begin{abstract}
The issue of target drift prediction based on ocean current from high-frequency (HF) ground-wave radar was studied in this paper. A large number of unpowered drift observation experiments for a class of South China Sea characteristic offshore fishing vessel was carried out in the range of radar radiation. The calculation of sub-grid velocity in the test area was based on the comparison between the HF radar data and the meteorological observation data of the buoy at sea. The two observation data also have good consistency in the matching of time and space. To analyze the temporal characteristics of diffusion velocity, the sample autocorrelation and the partial autocorrelation function of a sub-grid velocity were calculated separately, and a subgrid model based on the ARMA model was proposed. Firstly, trajectory simulation based on the Runge-Kutta method was used to preliminarily verify the improvement of target drift prediction accuracy by ARMA model. In addition, the Monte-Carlo method was used to calculate and compare the prediction range of different sub-grid velocity models, and the ARMA model with different continuous prediction time steps was studied. Compared with the traditional sub-grid velocity model, the ARMA model with short-term continuous prediction can significantly improve the performance of target
\end{abstract}


drift prediction model in terms of mean prediction error and separation. However, ARMA model is also limited by its continuous prediction step size to a large extent. On the one hand, this study verifies that the ARMA model with short-term continuous prediction improves the performance of target drift prediction model. On the other hand, the results further demonstrated that measured data provided by the HF radar, combined with other continuous ocean observation data, are of value for trajectory analysis of drift targets.

Key words: Target Drift Prediction; Search and Rescue; Sub-grid scale velocity model; Diffusion; High-Frequency Radar

\section{Introduction}

The development and utilization of marine resources have become the strategic focus of the development of countries all over the world. However, With the increasingly frequent maritime activities, it is difficult to avoid the threat of marine disasters in these areas with intensive human activities, especially in some areas seriously affected by disasters such as storm surges and tsunamis. Many marine incidents (Liu et al., 2015; Rusu, L., 2010; Deng et al., 2013).) such as like shipwrecks, oil spills and other navigation safety and environmental pollution accidents caused by these marine disasters occurred in the world's various seas every year. The 2011 Fukushima nuclear disaster, triggered by a tsunami in the Northeast Pacific Ocean, released large amounts of radioactive material into the sea. These radioactive materials drift and spread with the ocean current, causing immeasurable ecological damage to the surrounding sea ( $\mathrm{Lu}$ et al., 2021). Taking the South China Sea region as an example, the coastal areas of Guangdong Province have a high frequency of marine navigational accidents every summer, especially under typhoon weather. To deal with these marine accidents and disasters, it is necessary to establish an effective maritime emergency handling mechanism. Target drift prediction is the basis of marine emergency work, especially in maritime search and rescue, oil spill and contamination diffusion events, 
the accuracy and timeliness of drift prediction is the prerequisite for the success of emergency work. To avoid the blindness of operation organization and improve the efficiency and accuracy of maritime rescue command and coordination, it is very important to study the prediction model technology of maritime target drift.

The prediction of target drift at sea is a very complicated process. Firstly, different targets have different motion mechanisms in response to the dynamic factors of the marine environment. Secondly, there are also many uncertainties in the simulation of the marine environment dynamics. In general, the research on the prediction of maritime target drift is mainly divided into two aspects: the drift motion modeling and the search area calculation of maritime targets. The drift motion modeling mainly simulates its trajectory by analyzing the response law of target drift motion to marine atmospheric environment dynamics, while the search area calculation of maritime target calculates the search area by analyzing the trajectory simulation error based on trajectory simulation.

Research on drift motion modeling has a long history. Among them, the AP98 leeway model and drifting dynamics model are two common models. The latter one is mainly based on simplified dynamic analysis while the former one combines statistics and laws of physics. And both models require large amounts of experimental data to calibrate model parameters for different drift targets. In contrast, the simulation of the AP98 leeway model is more refined and has more applications (Zhu et al., 2019). Many research institutions have carried out a series of field observation experiments combined with the AP98 Leeway model, and the results have been applied to the corresponding search and rescue and oil spill emergency systems in more and more countries(Coppini et al., 2016; Breivik, O. and Allen, A.A., 2008; Brushett et al., 2017).

The Monte Carlo method based on Lagrangian particle tracking has been proved to be the most effective method for the search area calculation of maritime targets. The main challenge in the calculation process is the uncertainty in the marine environmental data. With the development of ocean numerical models, although the spatial and temporal resolution of ocean models has been refined, their numerical simulation 
accuracy still needs to be improved in the coastal areas where people are most active and concerned. In recent years, many researches (Barrick et al., 2012; Zelenke, B.C. 2005; Liu et al., 2014; Paduan, J.D. and Washburn, L., 2013) proved that highfrequency (HF) radar systems have become an alternative for the provision of accurate surface current maps in near-coastal environments. HF Radar is nowadays the only technology capable of providing real-time surface currents continuous in space and time for wide areas, from a few kilometers to up to $200 \mathrm{~km}$ offshore(Abascal et al., 2017).

Although the HF Radar system has great advantages in a real-time inversion of the ocean flow field, due to the limitation of its observation accuracy, it is still difficult to solve the problem of small-scale turbulence caused by complex topography and shoreline conditions. Given the influence of horizontal turbulence in the ocean flow field, most Monte Carlo method models currently use two kinds of sub-grid velocity models for approximate simulation: random walk model (De Dminicis et al., 2012) and more complex second-order random flight model (Spaulding et al., 2006). In the past, many studies used a simpler random walk model, which ignored the autocorrelation of the changes of the sub-grid velocity, such as the National Oceanic and Atmospheric Administration's (NOAA's) operational model for oil spills (Drouin et al., 2019; Beegle-Krause, C., 2001) and the Operational Search and Rescue Model for the Norwegian Sea and the North Sea. The random flight model takes into account the autocorrelation of the sub-grid velocity changes, In general, higher-order Markov models reproduce the observed statistics of ocean drifters better than random walk models (Griffa et al., 2007; Drouin et al., 2019). In the study of David S. Ullman (Ullman et al., 2006) and Kui Z (Zhu et al., 2021), The random walk model and the random flight model were further validated by the drifting buoy trajectory data combined with the Monte Carlo particle tracking simulation results.

In the past studies(Abascal et al., 2009; Shen et al., 2019; Abascal et al., 2012), there were many researches on buoy-type targets using HF Radar, but few on search and rescue targets. In addition, the velocity diffusion coefficient of the sub-grid in the study area was usually regarded as a constant in both the random walk model and the 
random flight model, the temporal and spatial variation of the diffusion coefficient in different areas was not taken into account. In addition, most of the relevant researches on the random walk model and the random flight model are aimed at some drifting buoys, rather than common search and rescue targets. The effects of wind on them are more complex. Therefore, the main research objective of this paper is to study the velocity model of the sub-grid combined with real potential targets in distress, learn the time variation of the velocity diffusion coefficient of the sub-grid, and verify its influence.

In this work, a class of offshore fishing vessels whose wind-induced drift coefficients have been obtained through a large number of observation experiments was taken as experimental targets for drift prediction. First, the obtained diffusion velocity was parameterized for two common sub-grid velocities, the model random walk model and the random flight model respectively. The time scales and diffusion coefficients of 2 components (the east-west and north-south) of the diffusion velocity were obtained. Then, the time series of diffusion velocity was further analyzed, and the optimal fitting ARMA model coefficients of the 2 diffusion velocity components were obtained by using the difference method and parameter calibration respectively. On this basis, this work focused on the target drift prediction model based on different sub-grid velocity models. Firstly, trajectory simulation based on the Runge-Kutta method was used to preliminarily verify the improvement of target drift prediction accuracy by ARMA model. In addition, the Monte-Carlo method was used to calculate and compare the prediction range of different sub-grid velocity models, and the ARMA model with different continuous prediction time steps was studied. Finally, the simulation results of target drift prediction models with different schemes were evaluated from two aspects of average prediction error and separation, and the validity of ARMA model based on short-term continuous prediction step size was verified. 


\section{Field Experiments}

\subsection{HF Radar Surface Currents}

In 2017, the three HF (High-Frequency) ground-wave radars observation system was built in Gaolan, Wanshan and Shangchuan respectively to conduct joint network observation of the marine environment in the Pearl River Estuary area area with the support of the National Key Research and Development Project. The radar uses a vertically polarized antenna to radiate electromagnetic waves to the surface. The electromagnetic waves interact with the rough sea surface, and the first-order scattering and second-order scattering will generate different amplitude peaks in the radar sea surface echo spectrum. Based on this principle, sea state information can be extracted from the radar echo spectrum.

Radial velocities observed at each of the 3 sites were obtained with the Multiple Signal Classification (MUSIC) algorithm (Schmidt, R.O. 1986) using measured antenna patterns. The vector velocity in the common radiation area of the three radar stations can be synthesized from the radial velocity in different directions. The time resolution of the radar inversion current field is 10 minutes, and the spatial resolution is $3 \mathrm{~km}$. The drift experiments in this paper were carried out in the Pearl River Estuary of the South China Sea, also in the common radiation area of the radar of the three stations. The latitude and longitude of the experiment range are $112.4^{\circ} \mathrm{E}-$ $114^{\circ} \mathrm{E}, 21.2^{\circ} \mathrm{N}-22.2^{\circ} \mathrm{N}$, which is shown in Figure.1. 


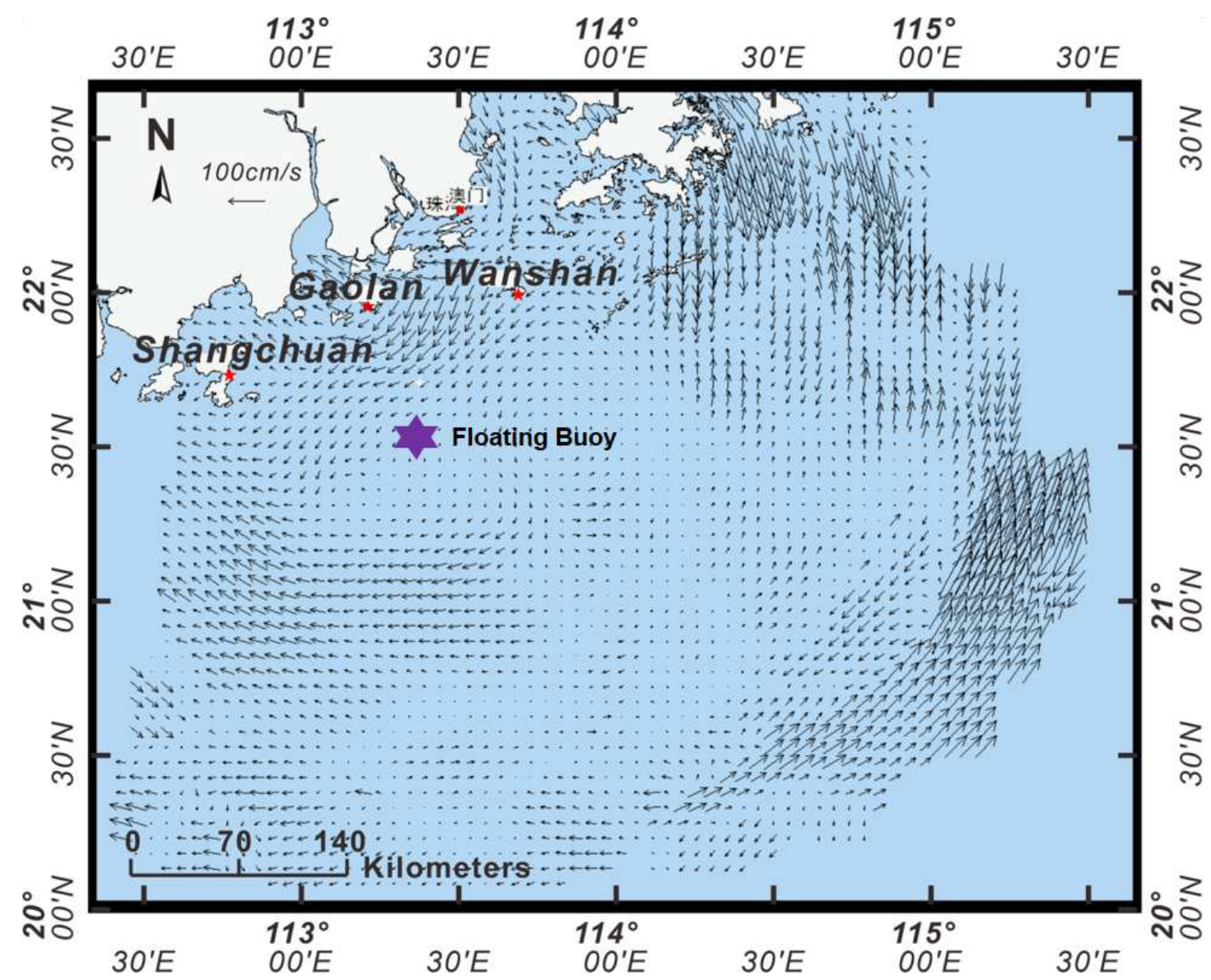

Figure. 1 Typical surface current map produced by CODAR showing the coverage area of the radar. The red star in the figure indicates the positions of the three stations HF ground-wave radar.

The red box represents the unpowered drift experimental area of the offshore fishing vessel.

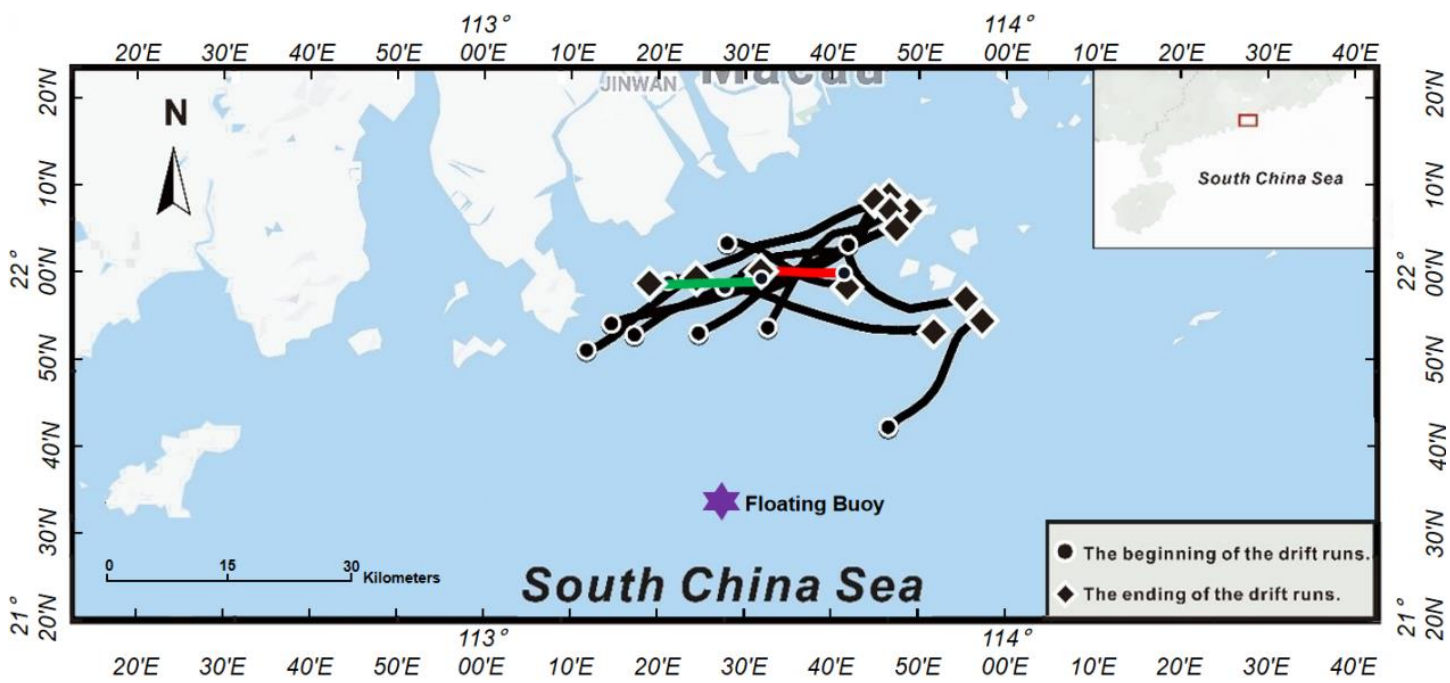

Figure. 2 Trajectories of the fishing vessel. The black trajectories represent the experiments conducted From November 7, 2018 to December 2, 2018, while the green one and the red one represent the experiments conducted in 2021.

\subsection{Drift Object}

Field experiments are specifically aimed at popular Chinese offshore fishing vessels 
in South China Sea. More precisely, the total length of the offshore fishing vessel is $26.3 \mathrm{~m}$, the design draft is $2.5 \mathrm{~m}$ and the gross tonnage is 121 tons. Due to the large number and common occurrence of such fishing vessels in the Pearl River Estuary, we carried out a series of field tracking and observation experiments for such fishing vessels from 2018 to 2021. From November 7, 2018 to December 2, 2018, the experiment lasted for about a month. Figure. 2 shows the drift trajectory of the experimental fishing boat, and Figure.3 shows its shape in detail. During the experiments, the offshore fishing vessel was outfitted with various instruments to observe the values (location, current velocity and wind velocity) of concern, including a GPS receiver, ADCP current meters, and a weather station. The observation frequency of all instruments was set at $1 \mathrm{HZ}$. According to this work, we obtained the windinduced drift coefficient of this type of fishing vessel combined with the AP98 model, as shown in Table.1(Zhu et al., 2019).

Table.1 The AP98 model leeway parameters.

\begin{tabular}{cccc}
\hline & Slope $(\%)$ & $\mathrm{Y}(\mathrm{cm} / \mathrm{s})$ & $\mathrm{S}_{\mathrm{yx}}(\mathrm{cm} / \mathrm{s})$ \\
\hline $\mathrm{DWL}$ & 4.39 & -4.56 & 7.01 \\
\hline$+\mathrm{CWL}$ & 2.18 & 2.25 & 6.60 \\
\hline$-\mathrm{CWL}$ & -0.57 & -4.29 & 4.16 \\
\hline
\end{tabular}

In 2021, in order to compare the observation data of HF radar network and verify the application of HF radar inversion current field in the prediction of target drift, we laid an additional floating buoy in this area, the longitude and latitude are $113.4537^{\circ} \mathrm{N}$ and $21.5612^{\circ} \quad \mathrm{E}$. Later, we conducted an unpowered tracking experiment again in this sea area for such Chinese offshore fishing vessels.

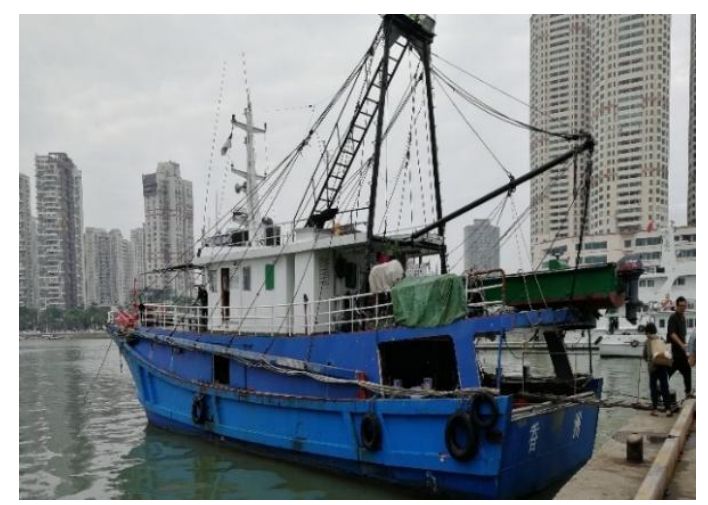

Fig. 3 Typical Chinese offshore fishing vessel. 


\section{3、Methodology}

\subsection{Lagrangian Particle Tracking Model}

The lagrangian particle tracking model is the most practical method for simulating target drift motion at sea. It has been widely used in various fields, including maritime search and rescue, oil spills, marine plankton, and sea ice(Zhang et al., 2017; Rixen, M. and Ferreira-Coelho, E. 2007; Rabatel et al., 2018). In general, the movements of these targets are mainly affected by surface current, wind, wave, and turbulent diffusion. Different target types are affected differently. This transport is governed by the transport equation in the Lagrangian form:

$$
\frac{d x}{d t}=L(x, t)+U_{F c}(x, t)+U_{F w}(x, t)
$$

Where, $\mathrm{x}$ represents the position of the floating target on the sea surface, $L$ and $U_{F w}$ respectively represents the drift velocity caused by wind and wave, and $U_{F c}$ represents the drift velocity caused by the current. According to Allen(Allen et al., 1999, 2010) and previous studies, for small targets (less than $30 \mathrm{~m}$ ), wave forces can be ignored, and wave-induced drift velocity is generally considered to be included in windinduced drift velocity.

For wind-induced drift velocity, the AP98 model(Allen et al., 1999, 2010; Breivik et al., 2011) with higher complexity was adopted in this work. In the AP98 model, windinduced drift velocity is decomposed into downwind leeway (DWL) drift velocity and crosswind leeway (CWL) drift velocity:

$$
\begin{cases}L_{d}=a_{d} U_{w}+b_{d}+\varepsilon_{d} & (D W L) \\ L_{c+}=a_{c+} U_{w}+b_{c+}+\varepsilon_{c+} & (+C W L) \\ L_{c-}=a_{c-} U_{w}+b_{c-}+\varepsilon_{c-} & (-C W L)\end{cases}
$$

Here, $L_{d}$ is the downwind leeway, which is related to the wind speed $U_{\text {wind }}$, the slope $a_{d}$, the offset $b_{d}$, and the error term ${ }^{\varepsilon_{d}}$. The same linear regression can be performed for both the positive CWL $\left(L_{c+}\right.$, right of the downwind) and the negative CWL ( $L_{c-}$, left of the downwind) crosswind directions. $U_{\text {wind }}$ is the wind speed at a 
height of $10 \mathrm{~m}$ on the sea surface. In this work, the wind speed was from the Weather station carried by the experimental offshore fishing vessel.

The current-induced drift velocity $U_{F c}$ is generally considered to be approximately equal to the surface current velocity $U_{c}$, which can be expressed as:

$$
U_{c}(x, t)=U_{a}(x, t)+U_{s}(x, t)
$$

Where, $U_{a}$ and $U_{s}$ represent advection velocity and diffusion velocity respectively. Advection velocity $U_{a}$ can be provided from velocity retrieved by HF radar. The diffusion velocity $U_{s}$ is mainly caused by some unsolved turbulent velocity, which is also referred to as sub-grid velocity in ocean numerical simulation. It should be pointed out that the advection velocity $U_{a}$ provided by HF radar inevitably includes some observation errors, but radar observation errors are usually difficult to separate from these unsolved turbulent velocities, so we include these velocities in the influence by referring to David S. Ullman.

\subsection{Sub-grid model}

\subsubsection{Random Walk Model and Random Flight Model}

There are two major categories of the sub-grid model in the past studies: random walk model and random flight model. These two models assume that the two components of the velocity at any point in the velocity field are independent of each other. In addition, it is also assumed that the particle position is a Markov process, and the random flight model also assumes that the velocity of the particle is a Markov variable. Physically, the random-flight model recognizes that the turbulent velocity fluctuations have a finite temporal correlation scale whereas the random-walk model assumes that the correlation scale is infinitesimal.(Ullman et al., 2006) In general, when the variance of diffusion velocity is the same, the particle diffusivity with the random flight model is larger.

Under the assumption of the random walk model, the equations that describe the diffusion velocity for a single component can be written as 


$$
d x=U_{a} d t+d s
$$

$$
d s=\left(K_{w}\right)^{1 / 2} d w
$$

$$
K_{w}=\sigma^{2} T
$$

In the above formulas, $d x$ represents the the total displacement of the particle during the time dt, it can be decomposed into displacements caused by advection and displacements caused by diffusion $d s . \mathrm{dw}(\mathrm{t})$ is a normal distributed random variable with a mean of zero, and its second moment is $\langle d w \cdot d w\rangle=2 \cdot d t . K_{w}$ is the diffusion coefficient and its value depends mainly on the standard deviation of diffusion velocity $\sigma$ and diffusion time scale $T$. The diffusion time scale $T$ in (6) must be $T=d t / 2$ in order to have the correct standard deviation of diffusion velocity.

Under the assumption of the random-flight model, the equations that describe the diffusion velocity for particle motion can be written as velocity to be correlated at different time steps. It could be found from first term on the

Differ from the ramdom walk model, the ramdom flight model assumed the diffusion right of (8), the change of advection velocity $U_{a}$ has a certain memory. $U_{s}(0)=u$, it can be drawn from Gaussian distribution with mean zero and variance $\mathrm{u}$. $\mathrm{Kf}$ is the diffusion coefficient and its value depends mainly on the standard deviation of diffusion velocity $\sigma$ and diffusion time scale T. $\mathrm{dw}(\mathrm{t})$ has the same meaning as above.

\subsubsection{ARMA Model}

In the past studies of both the random flight model and the random flight model, the diffusion coefficients they used were usually treated as constants in different regions and at different times. Zhu et al. used a large number of drifting buoy data to simulate and study the diffusion coefficient in different areas of the West Coast of the United 
States, but there is still a lack of studies on its temporal variation.

ARMA model is an important method to study time series, which is composed of autoregression model (AR model) and moving average model (MA model). Different from the above two sub-grid velocity models, the ARMA model forecasts the current and future target values by using the historical values continuously observed in the past. ARMA model has better performance in mining the internal relationship of data, but it also has high requirements on the continuity of observation data in its application. Meanwhile, the ARMA model is also one of the high-resolution spectral analysis methods of the parametric method, which is suitable for some data processing with periodic and random mixed signals. Considering that diffusion velocity $U_{s}$ contains turbulence with a certain autocorrelation time scale and random observation errors, a method to simulate the sub-grid velocity time series $\left\{U_{s}^{t}\right\},(t=1, \ldots, n)$ directly by using the ARMA model, avoiding diffusion coefficient simulation was tested. First, $U_{s}^{t}$ was decomposed into the east-west and north-south directions respectively:

$$
U_{s}^{t}=\left(U_{s x}^{t}, U_{s y}^{t}\right)
$$

The ordinary ARMA $(p, q)$ model requires the marginal distribution associated with the stochastic process under study to be normally distributed, which may not be the case for $U_{s x}^{t}$ and $U_{s y}^{t}$. Therefore, $\operatorname{ARIMA}(\mathrm{p}, \mathrm{n}, \mathrm{q})$ model was adopted in this work to make an n-order difference for $U_{s x}^{t}$ and $U_{s y}^{t}$ respectively, and obtain their stationary time series $\hat{U}_{s x}^{t}$ and $\hat{U}_{s y}^{t}$ :

$$
\begin{aligned}
& \hat{U}_{s x}^{j}=\sum_{j}^{p} \phi_{x}^{j} \hat{U}_{x}^{t-j}+\varepsilon_{x}^{t}-\sum_{i}^{q} \theta_{x}^{i} \varepsilon_{x}^{t-i} \\
& \hat{U}_{s y}^{j}=\sum_{j=1}^{p} \phi_{y}^{j} \hat{U}_{y}^{t-j}+\varepsilon_{y}^{t}-\sum_{i=1}^{q} \theta_{y}^{i} \varepsilon_{y}^{t-i}
\end{aligned}
$$

Where $\mathrm{p}$ and $\mathrm{q}$ represent the lagging order in the AR autoregression model and MA moving average model respectively. They are determined by the autocorrelation coefficient and partial autocorrelation coefficient of corresponding time series 
respectively, which is similar to the calculation of time scale in the random flight model.

\section{Model Calibration}

The AP98 model parameter calibration of the fishing vessel was based on the unpowered drift experimental data in 2018 which have been given above (please refer to Zhu et al., 2019 for details,). Next, the data used for trajectory simulation and drift range prediction are the drift experiment data added in April 2021, while the data used to calibrate the three sub-grid velocity models in this chapter are the observation data of ground wave radar and floating buoy before the drift experiment in 2021.

\subsection{Estimating Diffusion Velocity Statistics}

In many past studies (Ullman et al., 2006; Abascal et al., 2009), the current velocity from HF radars was considered to be advection velocity, while the true velocity could be derived from ocean observation buoys. In this work, although the buoy observation used was moored, the experimental fishing vessel was close enough to the floating buoy, and the unpowered drift region of the fishing vessel also was small. It could be assumed that the diffusion velocity $\left(U_{s}\right)$ of the fishing vessel experimental area is the difference between the current velocity $\left(U_{\text {radar }}\right)$ from HF radars and the velocity $\left(U_{c}\right)$ derived from the floating buoy:

$$
U_{s}(x, t)=U_{c}(x, t)-U_{\text {radar }}(x, t)
$$

In addition, the time resolution of the current velocity provided by the HF radar and the floating buoy is both 10 minutes. A total of 180 continuous samples ( 30 hours) were selected to analyze the diffusion velocity before the unpowered drift experiment. Due to the breakdown of the radar system before, the amount of data we used for modeling was limited, but it was enough for the calibration of the ARMA model, random walk, and random flight model.

First, we decompose $U_{c}$ and $U_{\text {radar }}$ respectively, and obtain the components of the two velocities in the east-west $(\mathrm{X})$ and north-south $(\mathrm{Y})$ directions respectively. As 
shown in Figure.4, it can be found that $U_{c}$ and $U_{\text {radar }}$ have a high degree of coincidence in the $\mathrm{X}$ direction, and there is a certain difference in the velocities of the two groups in the $\mathrm{Y}$ direction.
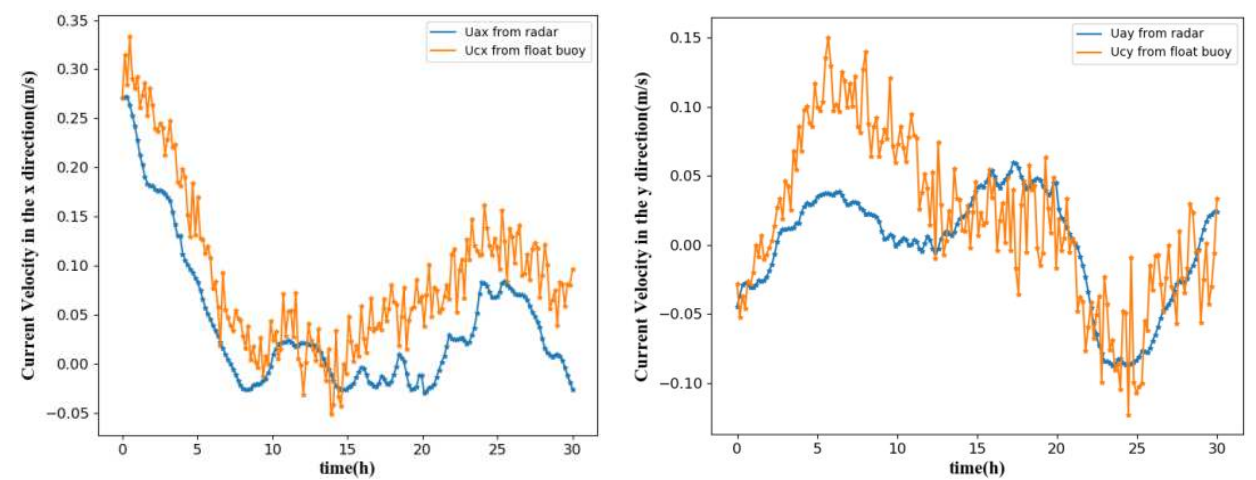

Figure.4 Radar and floating buoy observed velocity in the $\mathrm{X}$ (left) and Y(right) directions.

\subsection{Calculation of Diffusion Coefficients}

For the random walk and the random flight models, parameters $\sigma, K_{f}, T$ and $K_{w}$ are required to be solved by analyzing the obtained diffusion velocities. $\sigma$ represents the standard deviation of fitting diffusion velocity, which can be obtained directly. The diffusion scale $\mathrm{T}$ needs to be determined according to the autocorrelation function of $U_{s x}$ and $U_{s y}$ components respectively. For example, the h-order autocorrelation function (ACF) of $U_{s x}$ can be writen as:

$$
\rho(h)=\frac{\operatorname{Cov}\left(U_{s x}^{t}-U_{s x}^{t+h}\right)}{\sqrt{\operatorname{Var}\left(U_{s x}^{t}\right)} \sqrt{\operatorname{Var}\left(U_{s x}^{t+h}\right)}}=\frac{n^{-1} \sum_{t=1}^{n-h}\left(U_{s x}^{t}-\overline{U_{s x}}\right)\left(U_{s x}^{t+h}-\overline{U_{s x}}\right)}{\sigma_{U_{s x}}^{2}}
$$

In this work, the autocorrelation function of diffusion velocity was obtained by using samples with a 10-minute lag. To further estimate the time scale of diffusion velocity, we followed Ullman's (Ullman et al., 2006) method and used exponential function to fit the autocorrelation function of diffusion velocity optimally. 

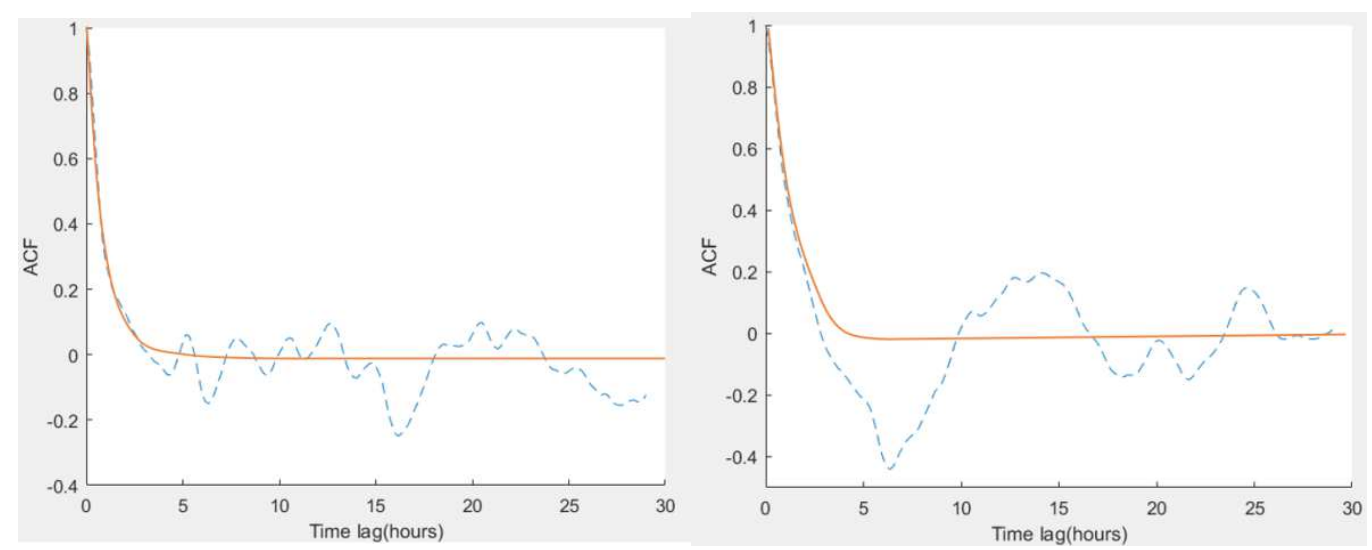

Figure.5 Lagged autocovariance functions of time series of the eastward diffusion component (left) and the northward diffusion component (right). The orange curves represent the obtained autocorrelation functions of diffusion velocities, while the blue curves represent the best fit exponential covariance functions.

Table.2 The optimal fitting parameters of the random walk model and the random flight model

\begin{tabular}{rrrrrr}
\hline$\sigma_{\mathrm{x}}(\mathrm{m} / \mathrm{s})$ & $T_{x}(\mathrm{~h})$ & $K_{\text {nxx }}\left(\mathrm{m}^{2} / \mathrm{s}\right)$ & $\sigma_{y}(\mathrm{~m} / \mathrm{s})$ & $T_{y}(h)$ & $K_{y y}\left(\mathrm{~m}^{2} / \mathrm{s}\right)$ \\
\hline 0.06 & 2.1 & 27.2 & 0.08 & 2.9 & 66.8 \\
\hline
\end{tabular}

In Figure.5, the two dotted lines are ACF curves of $U_{s x}$ and $U_{s y}$ components respectively, and the solid lines represent the optimal fitting exponential function. The ACF values of the two components declined rapidly and fluctuated between $[-0.2,0.2]$ after about a lag of 5 hours. All fitting parameters of the random walk and random model are shown in Table.2. The standard deviations of two diffusion velocity components, $\sigma_{\mathrm{x}}$, and $\sigma_{\mathrm{y}}$, are $0.05 \mathrm{~m} / \mathrm{s}$ and $0.07 \mathrm{~m} / \mathrm{s}$, and the time scales $\mathrm{T}_{\mathrm{x}}$ and $\mathrm{T}_{\mathrm{y}}$ are 2.1 $\mathrm{h}$ and $2.9 \mathrm{~h}$, respectively. It should be noted that only the value of $K_{w}$ was given in Table.2. For the random flight model, $K_{f}=K_{w} / T^{2}$.

\subsection{Calculation of ARMA Model Coefficients}

As is mentioned above, Ordinary ARMA $(p, q)$ model requires the marginal distribution associated with the stochastic process under study to be normally distributed, which may not be the case for $U_{s x}$ and $U_{s y}$. It can also be easily observed from Figure.4. Therefore, $U_{s x}$ and $U_{s y}$ should be differentiated first to smooth the 
sequence. In general, the modeling process of the ARMA model is as follows:

(1) To judge the data and determine whether it is a smooth and non-pure random sequence. If so, directly perform Step 2. If not, perform differential processing. Step 2 can proceed only after the marginal distribution associated with the stochastic process under study is normally distributed.

(2) Model recognition and order estimation through autocorrelation and partial autocorrelation functions.

(3) Parameter evaluation of the model.

(4) To test the fitting model for adaptability. If the model fails to pass the test, model recognition and order estimation will be performed again.

The smooth time series $\hat{U}_{s x}$ and $\hat{U}_{s y}$ were obtained after the first-order difference of $U_{s x}$ and $U_{s y}$, and the autocorrelation function of the corresponding time series has been given by Formula.14. Partial autocorrelation functions could be solved referring to the Yule-Walker equation(Wei, W., 2006). The autocorrelation function and partial autocorrelation functions of $\hat{U}_{s x}$ and $\hat{U}_{s y}$ components are shown in Figure.6 and Figure.7. The blue line in the figure represents $95 \%$ confidence interval. It can be found that from the figure that the autocorrelation coefficient and partial autocorrelation coefficient of the two components are gradually attenuated. The autocorrelation coefficient of the two components are both within the $95 \%$ confidence interval after the lag of 5 orders, and the partial autocorrelation coefficients are both within the $95 \%$ confidence interval after the lag of 6 orders. Therefore, for $U_{s x}$ and $U_{s y}$, the order of the model were both determined to be $\operatorname{ARIMA}(6,1,5)$. 

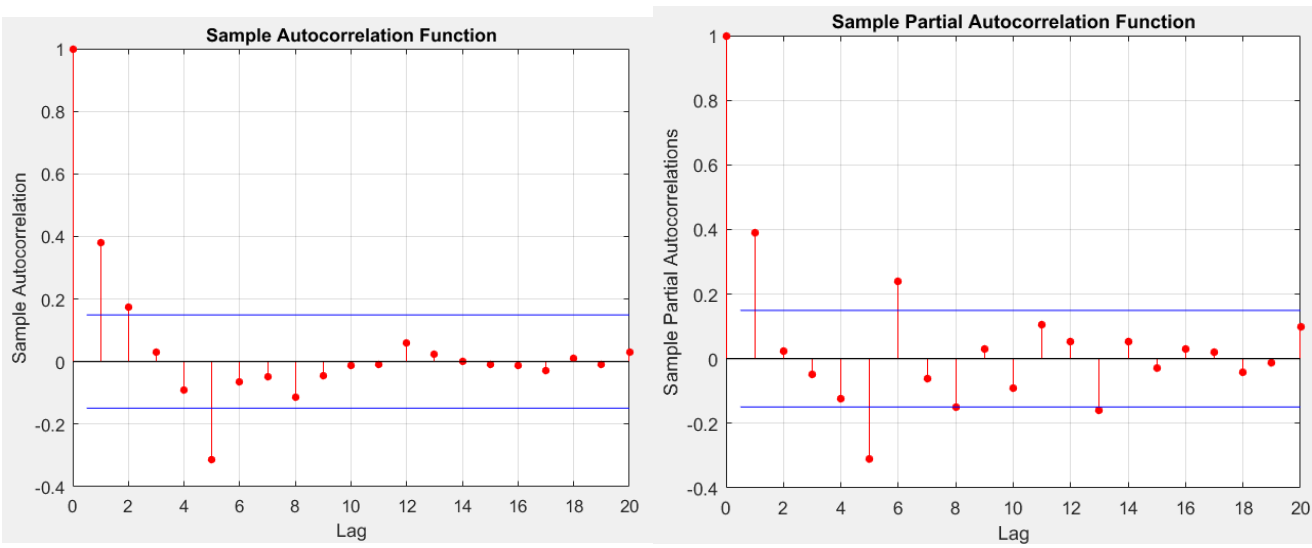

Figure.6 The sample autocorrelation function and the partial autocorrelation function of $U_{s x}$
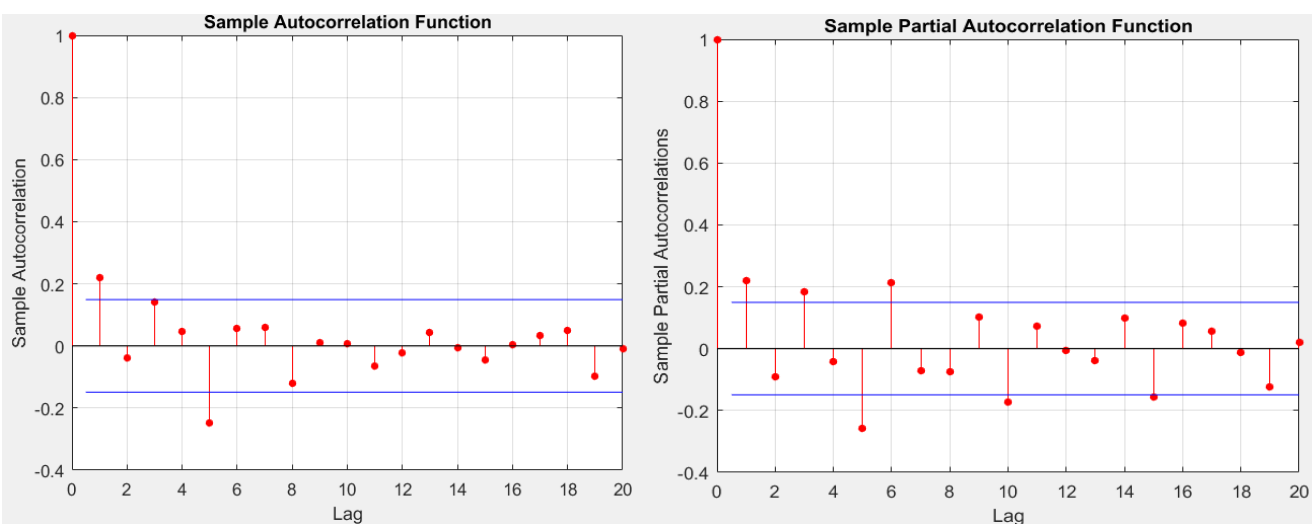

Figure.7 The sample autocorrelation function and the partial autocorrelation function of $U_{s y}$

For $U_{s x}$ and $U_{s y}, \operatorname{ARIMA}(6,1,5)$ model coefficients are shown in Table.3. $\sigma_{x \varepsilon}$ and $\sigma_{y \varepsilon}$ represent the standard deviation estimates of the residuals. The hypothesis that there is no correlation between the residuals after the establishment of ARIMA $(6,1,5)$ model was tested. The $\mathrm{p}$ values obtained are $(0.76$ and 0.68$)$ for $U_{s x}$ and $U_{s y}$ residuals respectively under the $5 \%$ significance level, it shows the established model has pass the hypothesis test.

Table. 3 The optimal fitting parameters of the $\operatorname{ARIMA}(6,1,5)$ model

\begin{tabular}{lllllllllllll}
\hline Parameter & $\phi_{x}^{1}$ & $\phi_{x}^{2}$ & $\phi_{x}^{3}$ & $\phi_{x}^{4}$ & $\phi_{x}^{5}$ & $\phi_{x}^{6}$ & $\theta_{x}^{1}$ & $\theta_{x}^{2}$ & $\theta_{x}^{3}$ & $\theta_{x}^{4}$ & $\theta_{x}^{5}$ & $\sigma_{x \varepsilon}$ \\
\hline Value & - & 0.25 & - & - & - & 0.03 & 0.47 & - & 0.53 & - & 0.18 & 0.03 \\
& 0.07 & & 0.51 & 0.02 & 0.68 & & & 0.23 & & 0.16 & & \\
\hline Parameter & $\phi_{y}^{1}$ & $\phi_{y}^{2}$ & $\phi_{y}^{3}$ & $\phi_{y}^{4}$ & $\phi_{y}^{5}$ & $\phi_{y}^{6}$ & $\theta_{y}^{1}$ & $\theta_{y}^{2}$ & $\theta_{y}^{3}$ & $\theta_{y}^{4}$ & $\theta_{y}^{5}$ & $\sigma_{y \varepsilon}$ \\
& & & & & & & & & & &
\end{tabular}




\begin{tabular}{llllllllllllll}
\hline Value & 1.10 & 0.63 & - & 0.98 & - & - & - & - & 0.63 & 0.77 & 0.39 & 0.05 \\
& & & 0.63 & & 0.86 & 0.13 & 0.73 & 0.41 & & & & & \\
\hline
\end{tabular}

401

\section{Simulation and Comparison}

\subsection{Trajectory simulation}

The data used for verification and comparison in this paper is the unpowered drift trajectory experimental data on April 20,2021. The data is divided into two trajectories (from 5:58 to $11: 58$ and from 13:18 to $22: 18$ ) respectively. There was an interval of about 20 minutes between the two trajectories because the fishing vessel encountered other fishing boats operating in the area during drifting, and the experiment was interrupted.

First, The previously described Lagrangian particle tracking model was used to reproduce the trajectories of the fishing vessel during the study period. The treatment of wind-induced drift and current-induced drift is consistent with some previous studies in this work. For wave-induced drift, we followed Breivik and Allen (Breivik et al., 2011) and Zhang(Zhang et al., 2017) and argued that the Stokes drift is directly related to the wind speed and the wave force can be ignored, so the wave effects are contained in the regression of the leeway of the object for small targets.

The time step of the Lagrangian particle tracking model is $60 \mathrm{~s}$. The fourth-order Runge-Kutta horizontal interpolation (Breivik and Allen 2008; Scott, Ferry et al. 2012) of the current field was used to drive the particle in the calculation of the particle continuous trajectory. The wind speed data used here were obtained from weather stations carried by the fishing vessel.

Two schemes were used to simulate the trajectory of the fishing vessel. The first one was to directly use the velocity observed by HF radars as the flow-induced drift velocity, and the second one was to use the ARMA model to modify the velocity observed by radar and then to calculate the current-induced drift velocity to simulate the trajectory. 

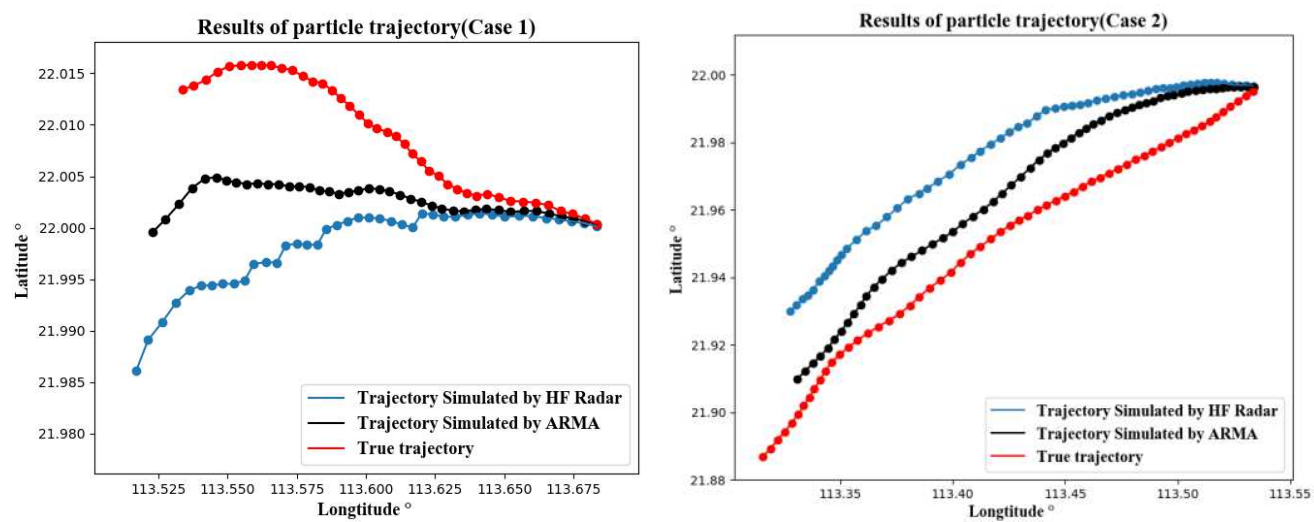

Figure. 8 Results of particle trajectory in the 2 cases. The true trajectory is plotted in red, while the trajectories simulated by HF radar and ARMA model are plotted in blue and black, respectively.

Simulation results of particle trajectory of the two cases based on the 2 schemes mentioned above are shown in Figure. 8. In Case 1, the simulation deviations of the two schemes were $3.8 \mathrm{~km}$ and $2.9 \mathrm{~km}$ after the 12 -hour calculation, while the actual total drift distance was $15 \mathrm{~km}$. In Case 2, the corresponding simulation deviations of the two models were $3.2 \mathrm{~km}$ and $1.6 \mathrm{~km}$, while the actual total drift distance was $25.5 \mathrm{~km}$. In the trajectory simulation of the 2 cases, the trajectory simulated by the ARMA model is significantly closer to the real trajectory.

\subsection{Predicted area calculation}

It can be obtained that the accuracy of target drift prediction by using ARMA model has been improved to some extent from the verification experiment of trajectory simulation. However, considering that there are too many uncertain factors in the process of target drifting at sea, it is necessary to adopt Monte Carlo method to simulate and further verify the trajectory set. In this work, We used 1000 particles for simulation, and disturbed the correlation coefficients of particle motion models including random walk model, random flight model, ARMA model, and AP98 model respectively.

The prediction range obtained at different times is determined by the spatial distribution of the 1000 particles. Convex hull algorithm or kernel density estimation method ( Martinez, W.L., Martinez, A.R., 2002) is usually used to estimate the prediction range. The kernel density estimation method was adopted in this paper. Its advantage lies in that it can calculate the distribution range of particles under different 
confidence intervals and give the region with large particle distribution density. Figure. 9 shows the predicted range with a confidence interval of $95 \%$ for the Case. 1

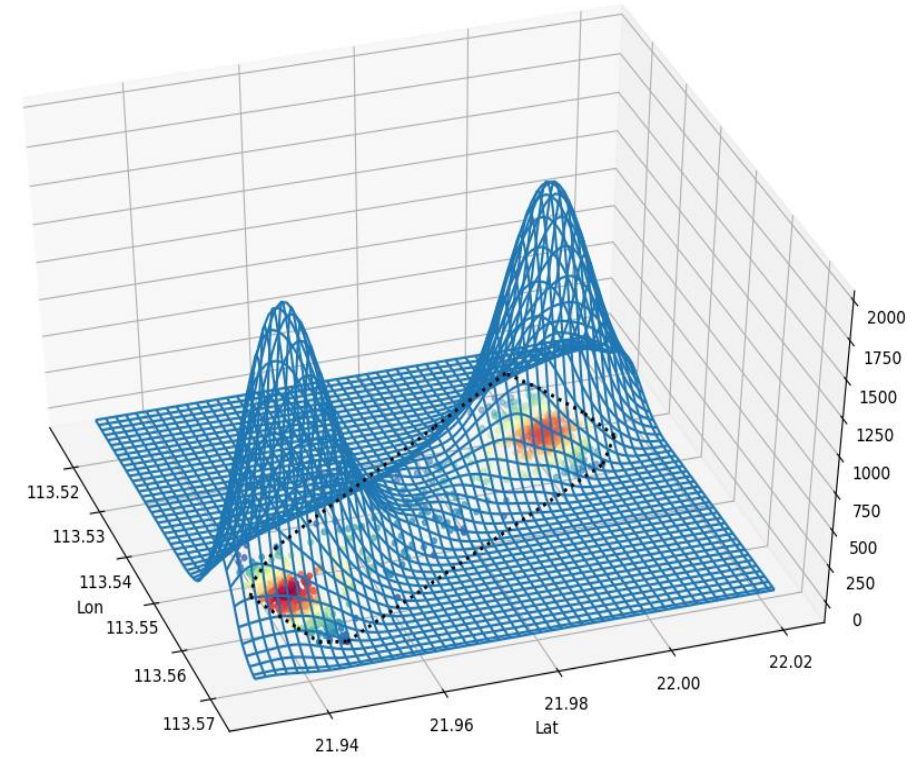

Figure 9 The predition area calculated by Kernel density estimation. The colorful dots represent the particle positions, the warmer the color, the more concentrated the particles. The black dotted line represents the predicted area of the $95 \%$ confidence level

5.2.1 Predicted area from the random walk model and the random flight model The prediction regions obtained by random walk model and the random flight model for the two cases of drift samples are shown in Figure.10. The simulated trajectories are indicated by gray lines. The simulated areas that represent a confidence interval of $95 \%$ are plotted in black dotted lines. The red dots represent the areas with concentrated particle distribution. It can be drawn from Figure.10 that in the simulation of each group of experiments, the regions with a relatively large probability density of prediction regions are distributed on both sides, which is mainly influenced by the wind-induced drift simulation scheme (AP98 model). The probability of the particle wind-induced drift direction is to the left or right of the wind was set at $50 \%$ each at the start of the calculation, and there is a chance of $3.1 \%$ for them to 'Jibing' to another side(Breivik et al., 2011). 

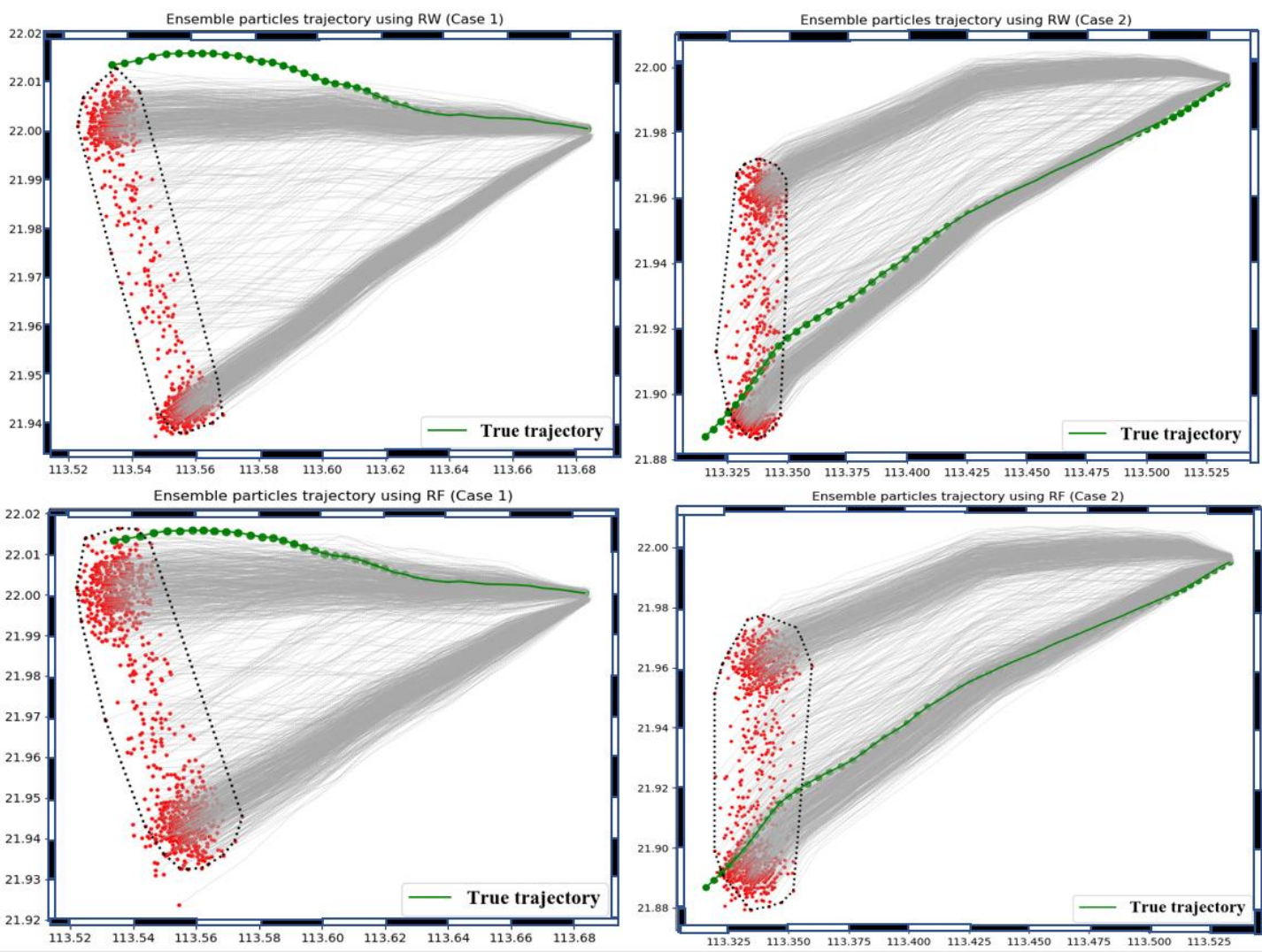

Figure.10 The predicted area from the random walk model and the random flight model for the 2

cases. The gray dotted line indicates the predicted range, and the red dots indicate the predicted final particle positions.

The trajectories of both cases generally moved from east to west. The simulation results shows that both the two sub-grid velocity models can simulate the drift prediction range of the target well, and the range of simulation is significantly increased over time. For the first case, we conducted a continuous 6-hour drift prediction. In the first three hours, the prediction regions obtained from the 2 groups of simulation schemes covered the real trajectory, but in the 4th-6th hours, the prediction regions obtained by the random walk model deviated from the real trajectory. For the second case, we conducted a continuous 9-hour drift prediction. The predicted region obtained from the 2 groups of simulation schemes was in good agreement with the real trajectory, but the predicted region obtained after 7 hours slightly deviated from the real trajectory.

\subsubsection{Predicted area from the ARMA model}

The ARMA model has good performance for some short-term forecasts, but for operational Marine emergency decision-making, it is usually necessary to estimate the 
drift and diffusion range of the target over a period of time. Therefore, two key problems need to be solved when using ARMA model to predict search range. On the one hand,

492 it is necessary to find the optimal continuous prediction step size of ARMA model, on 493 the other hand, a balance between continuous prediction step size and prediction 494 accuracy is pivotal as well. The schematic diagram of single-step and multi-step prediction of ARMA model is shown as Figure.11. Single-step prediction means that each prediction uses historical observations to predict one step, while multi-steps prediction refers to making a prediction every $\mathrm{n}$ steps, and each prediction uses historical observations to predict $\mathrm{n}$ steps forward.
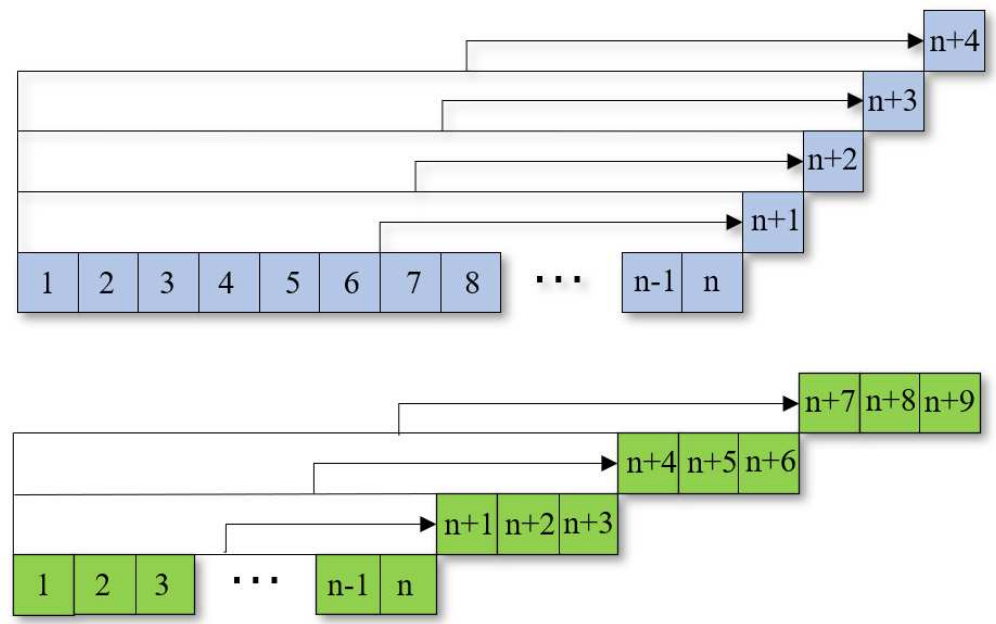

Figure.11 Single-step and multi-step prediction based on ARMA model
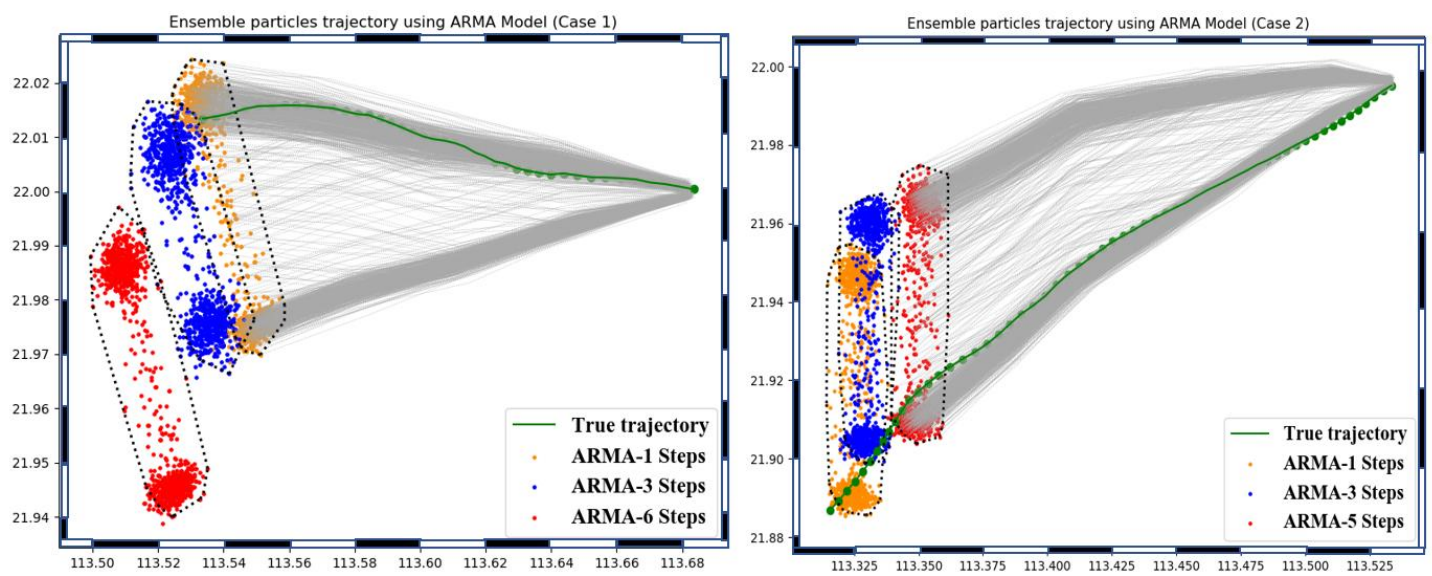

Figure.12 The predicted area from the ARMA model with different continuous predition time step for the 2 cases. The gray dotted line indicates the predicted range, and the dots with different colors indicate the predicted final particle positions. 
Figure. 12 shows the prediction regions calculated by ARMA models with different prediction step sizes. The gray lines represent the predicted trajectories of 1,000 particles. The dots in different colors in the figure represent the prediction results obtained by using different continuous prediction steps. It can be preliminarily found that the prediction range obtained by the model gradually deviates from the real trajectory as the continuous prediction step increases. However, at the same time, there is no significant difference in the area of prediction surface obtained by different prediction step sizes, which also indicates that the prediction step size has no significant influence on the separation of prediction range.

\section{Discussion}

To further compare and verify the performance of the random walk model, the random flight model and the ARMA model with different continuous prediction step size schemes, we selected two indexes to evaluate the simulation results, they are mean prediction error and separation respectively. The mean prediction error represents the average difference between the simulated 1000 particles and the real position of the target at different times (the time interval adopted in this work is 1 hour). The separation is the standard deviation of mean prediction errors. The separation represents the dispersion degree of predicted particles in spatial distribution. The larger the separation is, the larger the prediction area obtained by the model is. The combination of these two indexes above can evaluate the performance of the target prediction model to a certain extent. The mean prediction errors of different groups of simulation schemes are shown as Table. 4 and Table. 5.

Table.4 The mean prediction errors of the different groups of simulations in Case 1.

\begin{tabular}{ccccccc}
\hline \multicolumn{7}{c}{ Average distance error $(\mathrm{km})$} \\
\hline RW & $1 \mathrm{H}$ & $2 \mathrm{H}$ & $3 \mathrm{H}$ & $4 \mathrm{H}$ & $5 \mathrm{H}$ & $6 \mathrm{H}$ \\
\hline RF & 0.42 & 1.02 & 1.75 & 2.63 & 3.68 & 4.63 \\
ARMA(1S) & 0.43 & 1.1 & 1.92 & 2.86 & 3.89 & 4.96 \\
ARMA(2S) & 0.41 & 0.84 & 1.42 & 2.08 & 2.71 & 3.42 \\
ARMA(3S) & 0.41 & 0.82 & 1.43 & 2.05 & 2.65 & 3.39 \\
& & 0.89 & 1.5 & 2.36 & 3.02 & 3.73
\end{tabular}




\begin{tabular}{lllcccc} 
ARMA(4S) & 0.43 & 0.91 & 1.56 & 2.53 & 3.32 & 4.22 \\
ARMA(5S) & 0.42 & 0.96 & 1.73 & 2.58 & 3.56 & 4.6 \\
ARMA(6S) & 0.44 & 1.01 & 1.8 & 2.7 & 3.78 & 4.95 \\
ARMA(7S) & 0.44 & 1.13 & 1.96 & 3.01 & 4.19 & 5.12 \\
\hline
\end{tabular}

529

Table. 5 The mean prediction errors of the different groups of simulations in Case 2.

\begin{tabular}{cccccccccc}
\hline & \multicolumn{7}{c}{ Average distance error (km) } \\
\hline & $1 \mathrm{H}$ & $2 \mathrm{H}$ & $3 \mathrm{H}$ & $4 \mathrm{H}$ & $5 \mathrm{H}$ & $6 \mathrm{H}$ & $7 \mathrm{H}$ & $8 \mathrm{H}$ & $9 \mathrm{H}$ \\
\hline RW & 0.33 & 0.97 & 1.33 & 1.91 & 2.47 & 2.86 & 3.2 & 3.72 & 4.15 \\
RF & 0.33 & 0.99 & 1.43 & 2.12 & 2.66 & 3.1 & 3.46 & 3.98 & 4.44 \\
ARMA(1S) & 0.32 & 0.87 & 1.09 & 1.59 & 1.97 & 2.39 & 2.71 & 3.21 & 3.55 \\
ARMA(2S) & 0.32 & 0.9 & 1.1 & 1.62 & 2.01 & 2.43 & 2.79 & 3.32 & 3.69 \\
ARMA(3S) & 0.33 & 0.92 & 1.2 & 1.68 & 2.26 & 2.59 & 2.91 & 3.56 & 3.97 \\
ARMA(4S) & 0.33 & 0.95 & 1.25 & 1.87 & 2.44 & 2.86 & 3.19 & 3.66 & 4.1 \\
ARMA(5S) & 0.34 & 1.12 & 1.46 & 2.25 & 2.72 & 3.23 & 3.79 & 4.14 & 4.56 \\
ARMA(6S) & 0.36 & 1.22 & 1.56 & 2.35 & 2.86 & 3.55 & 3.95 & 4.21 & 4.81 \\
ARMA(7S) & 0.37 & 1.29 & 1.76 & 2.48 & 3.01 & 3.76 & 4.2 & 4.44 & 5.16 \\
\hline
\end{tabular}

Firstly, the simulation results of two traditional sub-grid velocity models, random walk model and random flight model are analyzed. In case 1, the 6-hour mean prediction errors of the two models were $4.63 \mathrm{~km}$ and $4.96 \mathrm{~km}$, respectively. In case 2 , the average 9-hour prediction errors of the two models were $4.15 \mathrm{~km}$ and $4.44 \mathrm{~km}$, respectively. The prediction error of the random walk model is slightly smaller than that of the random flight model, but there is no significant difference. The mean prediction errors of the two models increased significantly over time.

For the ARMA model, the simulation results from 1-step prediction to 7-steps prediction for both 2 cases were given as Table. 4 and Table.5. It can be drawn from the 2 tables that, in general, the mean prediction error of ARMA model increases with the increase of continuous prediction step (except in Case.1, the result of continuous 2steps prediction is slightly better than that of 1 -step prediction). However, in the shortterm continuous prediction with less than 5 steps, the mean prediction error of ARMA model is smaller than that of random walk model and random flight model. It indicated that the application of ARMA model is largely limited to its continuous prediction step size. 

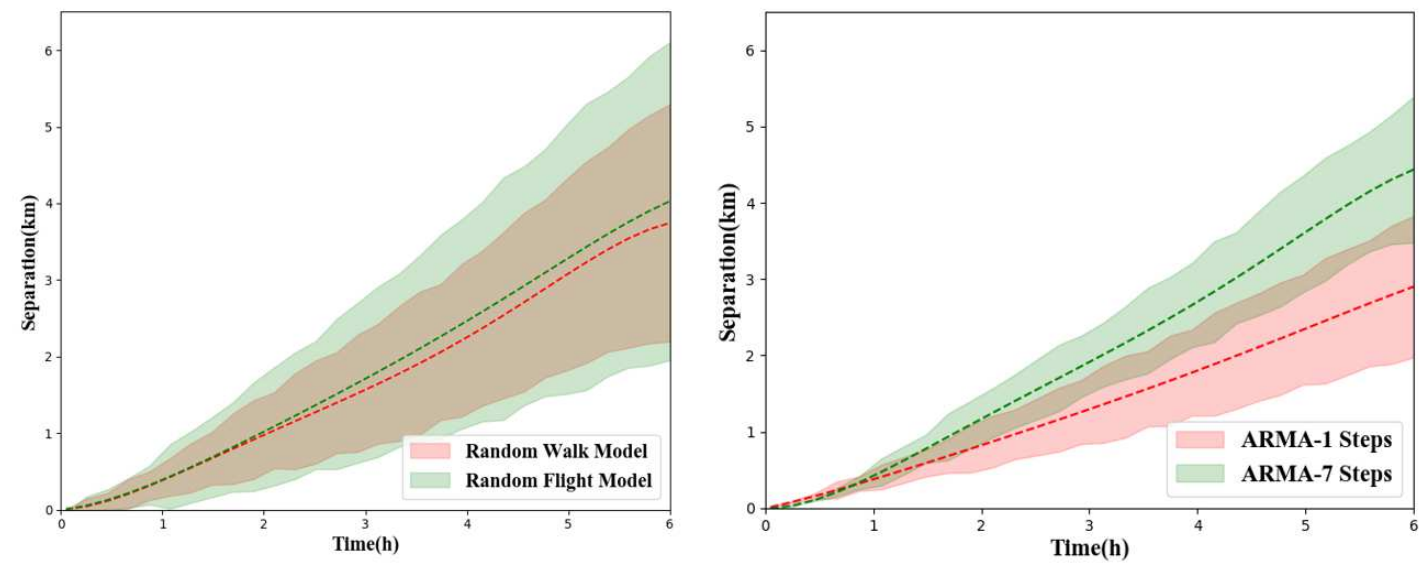

Figure.13 The mean predition errors and the separations for the different target predition schemes in 6 hours.

Figure.13 shows the separations of the calculated results of two classical subgrid velocity models and ARMA models with different predicted time steps in 6 hours. The dotted lines in the figure represent the mean prediction errors calculated by the target drift prediction model at different times, and the color-filled area represents its standard deviation, the separation. The mean prediction error and the separation present here was calculated using the mean of the 2 cases. Among the three models, the random flight model has the highest separation, followed by the random walk model, and the ARMA model has the lowest separation. Moreover, different predicted time steps have little influence on the separation of ARMA model simulation results. In addition, the average predicted areas of 3 groups of simulation schemes were also calculated for comparison. In Case 1, the 6-hour average predicted areas of the random walk model, random flight model, and ARMA(1-step) model are $22.3 \mathrm{~km}^{2}, 30.2 \mathrm{~km}^{2}$, and $17.9 \mathrm{~km}^{2}$, respectively; in Case 2, the 9-hour average predicted areas of 3 groups of simulation are $30.4 \mathrm{~km}^{2}$, $36.7 \mathrm{~km}^{2}$, and $28.2 \mathrm{~km}^{2}$, respectively. In the two cases, the predicted area based on the ARMA model with short-term prediction is the smallest which is consistent with the separation, while the predicted area based on the random flight model is the largest. In other words, the ARMA model with short-term prediction obtained the prediction range that is most consistent with the real trajectory and had the smallest area under the condition of the minimum average prediction error. The comparison of average predicted areas further demonstrates the advantages of the ARMA model over the two traditional sub-grid models. 


\section{Conclusions}

The issue of target drift prediction based on ocean currents from HF radars was studied in this work. The random walk model and the random flight model were calibrated and another sub-grid velocity model based on the ARMA model was proposed based on a large number of unpowered drift observation experiments for a type of offshore fishing vessel in the South China Sea within the radar radiation range and continuous observation data of floating buoys in the adjacent sea area. Based on the sub-grid velocity models, the drifts of the offshore fishing vessel were simulated and compared using the Lagrange particle tracking model and kernel density estimation algorithm.

The calculation of sub-grid velocity in the test area was based on the comparison between the HF radar data and the meteorological observation data of the buoy at sea. The two observation data also have good consistency in the matching of time and space. Firstly, trajectory simulation based on the Runge-Kutta method was used to preliminarily verify the improvement of target drift prediction accuracy by ARMA model. In addition, the Monte-Carlo method was used to calculate and compare the prediction range of different sub-grid velocity models, and the ARMA model with different continuous prediction time steps was studied. In the first case, the mean prediction error for 6 hours of the ARMA model(1-step) is $3.42 \mathrm{~km}$, and the area of the prediction range is $17.9 \mathrm{~km}^{2}$. In the second case, the mean prediction error for 9 hours of the ARMA model(1-step) is $3.55 \mathrm{~km}$, and the area of the prediction range is $28.2 \mathrm{~km}^{2}$, both of which are better than the random walk model and the random flight model. However, with the increase of prediction time step, the prediction accuracy of ARMA model decreases significantly. In the first case of experiments, when the continuous prediction time step is larger than 5 steps, the mean prediction error of the ARMA model exceeds that of the random walk model. In the second case of experiments, when the continuous prediction time step is larger than 4 steps, the mean prediction error of the ARMA model exceeds that of the random walk model. This also indicates that the application of ARMA model is largely restricted by the model's continuous prediction 
time step. In addition, the performance of the model was evaluated by the separation and the range of predicted area. The results show that different continuous prediction time steps have little influence on the separation of the simulated results of ARMA model, the predicted area obtained by the ARMA model is smaller than that obtained by random walk and random flight model most of the time.

The results indicate that the drift prediction model based on ARMA model with shortterm prediction has better performance than the random walk model and random flight model under the premise of sufficient HF radar observation velocity and other continuous observation data. However, the application of ARMA model is largely restricted by the model's continuous prediction time step. In addition, the ARMA model also has higher observation data requirements for operational target drift prediction work. On the one hand, the ARMA model relies on long-term continuous ocean observation data in the calculation area, but on the other hand, it also reflects the importance of floating buoys in operational marine emergency work, especially in areas with complex coastline topography, strong turbulence impact, and intensive human activities.

Although the validation has been performed using a fishing vessel, the results could be also applied for other floating objects or floating substances. The difference is that for oil slicks, some physical and chemical changes in their drift process need to be considered. This work also demonstrated that measured data provided by the HF radar, combined with other continuous ocean observation data, are of value for trajectory analysis of drift targets.

\section{Data availability}

All raw data can be provided by the corresponding authors upon request.

\section{Author contribution}

Kui Zhu: Conceptualization, Methodology, Software, Data curation, Writing original draft, Visualization. Lin Mu: Investigation, Supervision, Software, Validation. 
Haiwen Tu: Investigation, Supervision, Software, Validation. Runze Yu: Visualization, Writing - review.\& editing Xiaoyu Xia: Visualization, Writing - review.\& editing.

\section{Competing interests}

The authors declare that they have no conflict of interest.

\section{Acknowledgments}

This work was supported by grants from the National Key Research and Development Program of China (Grant No. 2017YFC1404700), National Natural Science Foundation of China (Grant No. U2006210), Shenzhen Fundamental Research Program (Grant No. JCYJ20200109110220482), and China Scholarship Council.

\section{References}

Abascal, A.J., Castanedo, S., Medina, R., Losada, I.J., and Alvarez-Fanjul, E.: Application of HF radar currents to oil spill modelling, Mar. Pollut. Bull., 58, 238-248, https://doi.org/10.1016/j.marpolbul.2008.09.020, 2009.

Abascal, A.J., Sanchez, J., Chiri, H., Ferrer, M.I., Cardenas, M., Gallego, A., Castanedo, S., Medina, R., Alonso-Martirena, A., Berx, B., Turrell, W.R., and Hughes, S.L.: Operational oil spill trajectory modelling using HF radar currents: A northwest European continental shelf case study, Mar. Pollut. Bull., 119, 336-350, https://doi.org/10.1016/j.marpolbul.2017.04.010, 2017.

Abascal, A.J., Castanedo, S., Fernandez, V., and Medina, R.: Backtracking drifting objects using surface currents from high-frequency (HF) radar technology, Ocean Dyn., 62, 1073-1089, https://doi.org/10.1007/s10236-012-0546-4, 2012.

Allen, A., and Plourde, J.V.: Review of Leeway: Field Experiments and Implementation, 199924 U. S. Coast Guard Research and Development Center, Groton, 351pp., 1999. Allen, A., Roth, J., Maisondieu, C., Breivik, O., and Forest, B.: Field Determination of 
the Leeway of Drifting Objects, The Norwegian Meteorological Institute, 43pp., 2010. Barrick, D., Fernandez, V., Ferrer, M.I., Whelan, C., and Breivik, O.: A short-term predictive system for surface currents from a rapidly deployed coastal HF radar network, Ocean Dyn., 62, 725-740, https://doi.org/10.1007/s10236-012-0521-0, 2012.

Beegle-Krause, C.: General NOAA oil modeling environment (GNOME): A new spill trajectory model, International Oil Spill Conference Proceedings, 865-871, https://doi.org/10.7901/2169-3358-2001-2-865, 2001.

Breivik, O., and Allen, A.A.: An operational search and rescue model for the Norwegian Sea and the North Sea, J. Mar. Syst., 69, 99-113, https://doi.org/10.1016/j.jmarsys.2007.02.010, 2008.

Breivik, O., Allen, A.A., Maisondieu, C., and Roth, J.C.: Wind-induced drift of objects at sea: The leeway field method, Appl. Ocean Res. 33, 100-109, https://doi.org/10.1016/j.apor.2011.01.005, 2011.

Brushett, B.A., Allen, A.A., King, B.A., and Lemckert, C.J.: Application of leeway drift data to predict the drift of panga skiffs: Case study of maritime search and rescue in the tropical pacific, Appl. Ocean Res., 67, 109-124, https://doi.org/10.1016/j.apor.2017.07.004, 2017.

Coppini, G., Jansen, E., Turrisi, G., Creti, S., Shchekinova, E.Y., Pinardi, N., Lecci, R., Carluccio, I., Kumkar, Y.V., D'Anca, A., Mannarini, G., Martinelli, S., Marra, P., Capodiferro, T., and Gismondi, T.: A new search-and-rescue service in the Mediterranean Sea: a demonstration of the operational capability and an evaluation of its performance using real case scenarios, Nat. Hazards Earth Syst. Sci. 16, 2713-2727, https://doi.org/10.5194/nhess-16-2713-2016, 2016.

De Dominicis, M., Leuzzi, G., Monti, P., Pinardi, N., and Poulain, P.M.: Eddy diffusivity derived from drifter data for dispersion model applications, Ocean Dyn., 62, 1381-1398, https://doi.org/10.1007/s10236-012-0564-2, 2012.

Deng, Z.G., Yu, T., Jiang, X.Y., Shi, S.X., Jin, J.Y., Kang, L.C., and Zhang, F.: Bohai Sea oil spill model: a numerical case study, Mar. Geophys. Res., 34, 115-125, https://doi.org/10.1007/s11001-013-9180-x, 2013. 
682 Drouin, K.L, Mariano, A.J., Ryan, E.H., and Laurindo, L.C.: Lagrangian simulation of 683 oil trajectories in the Florida Straits, Mar. Pollut. Bull., 140, 204-218, 684 https://doi.org/10.1016/j.marpolbul.2019.01.031, 2019.

685 Gao, J., Mu, L., Bao, X.W., Song, J., and Ding, Y.: Drift analysis of MH370 debris in 686 the southern Indian Ocean, Front. Earth Sci., 12, 468-480, 687 https://doi.org/10.1007/s11707-018-0693-0, 2018. Griffa, A.: Applications of stochastic particle models to oceanographic problems, R. Adler, P. Muller, B. Rozovskii (Eds.), Stochastic Modeling in Physical Oceanography, Birkhauser, Boston, 16pp., 1996.

691 Griffa, A., Kirwan, A., Mariano, A., Özgökmen, T., and Rossby, H.: Lagrangian Analysis and Prediction of Coastal and Ocean Dynamics, J. Atmos. Ocean. Technol., 19, 1114-1126, https://doi.org/10.1017/CBO9780511535901, 2007. Liu, X., Guo, J., Guo, M.X., Hu, X.K., Tang, C., Wang, C.Y., and Xing, Q.G.: Modelling of oil spill trajectory for 2011 Penglai 19-3 coastal drilling field, China, Appl. Math. Model., 39, 5331-5340, https://doi.org/10.1016/j.apm.2014.10.063, 2015. Liu, Y.G., Weisberg, R.H., and Merz, C.R.: Assessment of CODAR SeaSonde and WERA HF Radars in Mapping Surface Currents on the West Florida Shelf, J. Atmos. Ocean. Technol., 31, 1363-1382, https://doi.org/10.1175/JTECH-D-13-00107.1, 2014.

Lu Y, Yuan J, Du D, Sun B and Yi X: Monitoring Long-term Ecological Impacts from Release of Fukushima Radiation Water into Ocean, J. Geo Sus, https://doi.org/10.1016/j.geosus.2021.04.002, 2021. Mariano, A.J., Kourafalou, V.H., Srinivasan, A., Kang, H., Halliwell, G.R., Ryan, E.H., and Roffer, M.: On the modeling of the 2010 Gulf of Mexico Oil Spill, Dyn. Atmos.

Paduan, J.D., and Washburn, L.: High-Frequency Radar Observations of Ocean Surface Currents, Annu. Rev. Mar. Sci., 5, 115-136, https://doi.org/10.1146/annurev-marine121211-172315, 2013.

Rabatel, M., Rampal, P., Carrassi, A., Bertino, L., and Jones, C.K.R.T.: Impact of rheology on probabilistic forecasts of sea ice trajectories: application for search and 
rescue operations in the Arctic, Cryosphere., 12, 935-953, https://doi.org/10.5194/tc12-935-2018, 2018.

Rixen, M., and Ferreira-Coelho, E.: Operational surface drift prediction using linear and non-linear hyper-ensemble statistics on atmospheric and ocean models, J. Mar. Syst., 65, 105-121, https://doi.org/10.1016/j.jmarsys.2004.12.005, 2007.

Rusu, L.: Application of numerical models to evaluate oil spills propagation in the coastal environment of the black sea, J. Environ. Eng. Landsc. Manag., 18, 288-295, https://doi.org/10.3846/jeelm.2010.33, 2010.

Sayol, J.M., Orfila, A., Simarro, G., Conti, D., Renault, L., and Molcard, A.: A Lagrangian model for tracking surface spills and SaR operations in the ocean, Environ. Modell. Softw., 52, 74-82, https://doi.org/10.1016/j.envsoft.2013.10.013, 2014.

Schmidt, R.O.: Multiple emitter location and signal parameter-estimation, IEEE Trans. Antennas Propag., 34, 276-280, https://doi.org/10.1109/TAP.1986.1143830, 1986.

Shen, Y.T., Lai, J.W., Leu, L.G., Lu, Y.C., Chen, J.M., Shao, H.J., Chen, H.W., Chang, K.T., Terng, C.T., Chang, Y.C., and Tseng, R.S.: Applications of ocean currents data from high-frequency radars and current profilers to search and rescue missions around Taiwan, J. Oper. Oceanogr., 12 , S126-S136, https://doi.org/10.1080/1755876X.2018.1541538, 2019.

Spaulding, M., Isaji, T., Hall, P., and Allen, A.: A hierarchy of stochastic particle models for search and rescue (SAR): Application to predict surface drifter trajectories using HF radar current forcing, J. Mar. Environ. Eng., 8, 181-214,

Ullman, D.S., O'Donnell, J., Kohut, J., Fake, T., and Allen, A.: Trajectory prediction using HF radar surface currents: Monte Carlo simulations of prediction uncertainties, J. Geophys. Res.-Oceans., 111, https://doi.org/10.1029/2006JC003715, 2006.

Wang, J.H., and Shen, Y.M.: Modeling oil spills transportation in seas based on unstructured grid, finite-volume, wave-ocean model, Ocean Model. 35, 332-344, https://doi.org/10.1016/j.ocemod.2010.09.005, 2010.

Wei, W.: Time Series Analysis: Univariate and Multivariate Methods, 2nd edition, 605 pp., 2006. 
Zelenke, B.C.: An Empirical Statistical Model Relating Winds and Ocean Surface

741 Currents: Implications for Short-term Current Forecasts, Oregon State University, 2005.

742 Zhang, J.F., Teixeira, A.P., Soares, C.G., and Yan, X.P.: Probabilistic modelling of the 743 drifting trajectory of an object under the effect of wind and current for maritime search 744 and rescue, Ocean Eng., 129, 253-264, https://doi.org/10.1016/j.oceaneng.2016.11.002, 7452017.

746 Zhu, K., Mu, L., and Tu, H.W.: Exploration of the wind-induced drift characteristics of 747 typical Chinese offshore fishing vessels, Appl. Ocean Res., 92, 748 https://doi.org/10.1016/j.apor.2019.101916, 2019.

749 Zhu, K., Mu, L., and Xia, X.Y.: An ensemble trajectory prediction model for maritime 750 search and rescue and oil spill based on sub-grid velocity model, Ocean Eng., 236, 751 https://doi.org/10.1016/j.oceaneng.2021.109513, 2021. 